\title{
Kinetics characterization of c-Src binding to lipid membranes: switching from labile to persistent binding
}

\author{
Anabel-Lise Le Roux ${ }^{1,2}$, Maria Antònia Busquets ${ }^{3}$, Francesc Sagués ${ }^{4}$, Miquel Pons ${ }^{1 *}$
}

1. Biomolecular NMR Laboratory. Organic Chemistry Department. University of Barcelona. Baldiri Reixac 1012, 08028 Barcelona, Spain

2. Institute for Research in Biomedicine (IRB-Barcelona). Baldiri Reixac 10-12, 08028 Barcelona, Spain

3. Department of Physicochemistry. Faculty of Pharmacy. Nanoscience and Nanotechnology Institute (IN2UB). University of Barcelona. Joan XXIII 27-31, 08028 Barcelona, Spain

4. Department of Physical Chemistry. Faculty of Chemistry. University of Barcelona. Martí i Franquès 1,08028 Barcelona, Spain

* To whom correspondence should be addressed, email: mpons@ub.edu

\begin{abstract}
Cell signaling by the c-Src proto-oncogen requires the attachment of the protein to the inner side of the plasma membrane through the myristoylated N-terminal region, known as the SH4 domain. Additional binding regions of lower affinity are located in the neighbor intrinsically disordered Unique domain and the structured SH3 domain. Here we present a surface plasmon resonance study of the binding of a myristoylated protein including the SH4, Unique and SH3 domains of c-Src to immobilized liposomes. Two distinct binding processes were observed: a fast and a slow one. The second process lead to a persistently bound form (PB) with a slower binding and a much slower dissociation rate than the first one. The association and dissociation of the PB form could be detected using an anti-SH4 antibody. The kinetic analysis revealed that binding of the PB form follows a second order rate law suggesting that it involves the formation of c-Src dimers on the membrane surface. A kinetically equivalent PB form is observed in a myristoylated peptide containing only the SH4 domain but not in a construct including the three domains but with a 12-carbon lauroyl substituent instead of the 14-carbon myristoyl group. The PB form is observed with neutral lipids but its population increases when the immobilized liposomes contain negatively charged lipids. We suggest that the PB form may represent the active signaling form of c-Src while the labile form provides the capacity for fast $2 \mathrm{D}$ search of the target signaling site on the membrane surface.
\end{abstract}

\section{Highlights}

- Myristoylated c-Src bind to membranes in two forms kinetically distinguishable by SPR

- A persistently bound form of c-Src was studied by SPR using an anti-SH4 antibody.

- Formation of the persistently bound only requires the myristoylated SH4 domain.

- A fatty acid chain of at least 14 carbons is required for persistent binding.

- The persistently bound species is a c-Src dimer formed on the membrane.

\section{Keywords}

c-Src, Surface Plasmon Resonance; myristoylated proteins; intrinsically disordered proteins (IDP); protein dimerization on membranes; antibody capture; SH4 domains; Cell signaling; c-Src trafficking. 


\section{Introduction}

c-Src is the leading member of the Src family of non-receptor tyrosine kinases, (SFKs), which are involved in many signaling pathways [1] [2] [3]. The deregulation of these kinases, and in particular c-Src, affects cell migration, proliferation and survival [4]. Src is over expressed or up regulated in several tumor types, including colorectal (CRC) and prostate cancers [5] [2] and has been associated with the survival of bone metastasis breast cancer cells [6]. In addition, high activity levels of Src and the related kinase Yes have been linked to poor clinical prognosis [7] [8].

The N-terminal (SH4) region of c-Src is cotranslationally myristoylated at the N-terminal glycine residue and is rich in charged residues. The SH4 domain serves as a membrane-anchoring point. In its resting state, c-Src is localized near the perinuclear region and probably associated to endosomes [9], and is translocated to the cytoplasmic side of the plasma membrane upon stimulation [10]. Downstream signaling of c-Src depends on its sub cellular localization. c-Src activity causes RhoA inhibition at focal adhesion sites [2] but activation of the same protein at podosomes [11]. c-Src also participates in the formation of lamellipodia at the leading edge of migrating cells [12]. Proliferation and survival are mediated by c-Src through the RAS-MAPK and PIK3-Akt signaling pathways. The involvement of c-Src in such a variety of pathways reflects an exquisite spatial and temporal regulation of this molecule.

Most SFKs are both palmitoylated and myristoylated, but c-Src contains only one myristoyl group [13]. While the insertion of the single myristoyl chain provides barely enough hydrophobic energy to attach the protein to the lipid bilayer, concurrent hydrophobic and electrostatic interactions result in effective[14], although reversible, membrane anchoring[15]. A soluble pool of c-Src has been reported in several cell types (e.g. Cos-7, Swiss T3T cells) [16] [17] [18]. Fluorescence recovery after photobleaching of c-Src-GFP demonstrated the trafficking of c-Src between the plasma membrane and endosomes was fast and independent of actin and tubulin, thereby suggesting that c-Src trafficking involves short-term cytosolic release ("hopping") [15]. Non-vesicular transport has also been observed for non-palmitoylated Ras[19], although the recovery rate of Src-GFP fluorescence observed by Kashahara et al. [15] was slower than that of the non-palmitoylated Ras [19].

70 Surface plasmon resonance (SPR) is widely used for the characterization of the kinetics and thermodynamics of binding of macromolecules to immobilized receptors [20], lipids [21] or even entire cells [22]. Using SPR, here we show that cSrc anchoring involves two kinetically well-separated events, namely: a rapid reversible binding step followed by the formation of a persistently bound (PB) species. Kinetics modeling of the build-up of the slowly dissociating species is consistent with the formation of $\mathrm{c}-\mathrm{Src}$ dimers inserted in the membrane.

\section{Materials and Methods}

\section{Cloning, expression and purification}

The dual gene expression vector pETDuet-1 (Novagen), which comprises two cloning sites, was provided by Julian M. Glück [23]. The N-Myristoyl Transferase (NMT) gene was already introduced into the first site. The gene encoding the first 185 residues of c-Src, from now on called USH3 was cloned in the second site. USH3 comprises the SH4, Unique and SH3 domains, followed by a His6 purification tag.

USH3 protein expression was performed in Escherichia coli Rosetta $^{\text {TM }}$ (DE3)pLysS cells (Novagen). The protocol described by Glück et al. [23] gave a mixture of non-acylated forms, laurylated forms, myristoylated forms coeluting

85 with NMT and not separable from it, and a degraded form. To obtain pure myristoylated USH3 (MyrUSH3), we modified the protocol. Just before induction with isopropyl- $\beta$-D-thiogalactopyranosid (Melford) at final concentration of $1 \mathrm{mM}$, myristic and palmitic acid (Sigma) were added to the cell culture, at a final concentration of $200 \mu \mathrm{M}$. The cell pellet was resuspended in lysis buffer $(20 \mathrm{mM}$ TrisHCl, 300mM NaCl, 5mM Imidazole, pH 8), to which protease inhibitors were added (Protein Inhibitor Cocktail ${ }^{1}$, and $1 \mathrm{mM}$ PMSF, both from Sigma). Cells were sonicated and 90 centrifuged. The supernatant contained the previously described mixture, but pure MyrUSH3 was retained in the pellet, and subsequently extracted using lysis buffer supplemented with 1\% Triton X100 (Sigma). The non-acylated and degraded forms, which do not contain the myristoyl group, were not retained in the pellet. Further purification was performed in a Ni-NTA column (Qiagen) followed by size exclusion chromatography in a Superdex 75 26/60, in phosphate buffer $(50 \mathrm{mM} \mathrm{NaP}, 150 \mathrm{mM} \mathrm{NaCl}, 0.2 \mathrm{mM}$ EDTA, pH 7,5). The purity and identity of the product was established by HPLC and mass spectrometry (BioSuite pPhenyl 1000RPC 2.0 x $75 \mathrm{~mm}$ coupled to a LCT-Premier

1

Protein cocktail inhibitor: $23 \mathrm{mM}$ AEBSF, 1mM EDTA, $20 \mu \mathrm{M}$ Bestatin, $3 \mu \mathrm{M}$ Pepstatin A, $3 \mu \mathrm{M}$ E64 (final concentrations in lysis buffer) 
Waters from GE Healthcare).

Laurylated USH3 (LaurUSH3) was obtained as a side product during protein expression in E. coli as a result of the low selectivity of yeast NMT towards the shorter chain acyl CoA forms resulting from the $\beta$-oxidation of longer fatty acids. Given that palmitoyl CoA is not a good substrate of NMT but is metabolized to myristoyl CoA, the addition of palmitic acid reduced the undesired laurylation of the protein. LaurUSH3 was separated from MyrUSH3 by size exclusion chromatography, and its purity was verified by HPLC and MS.

Myristoylated SH4 (MyrSH4) was synthesized using standard solid-phase methods.

\section{Liposome preparation}

105 All lipids were from Tebubio. 1,2-Dimyristoyl-sn-glycero-3-phosphocholine (DMPC), 1,2-dioleoyl-sn-glycero-3phosphocholine, DOPC, and 1,2-dioleoyl-sn-glycero-3-phospho-(1'-rac-glycerol) (sodium salt), DOPG were dissolved in chloroform. 1,2-Dimyristoyl-sn-glycero-3-phospho-rac-(1-glycerol) sodium salt (DMPG) was dissolved in chloroform:methanol:H2O 65:35:8. Three lipid compositions were used, DMPC alone, DMPC:DMPG 2:1 or DOPC:DOPG 2:1. The dissolved lipids were evaporated in a rotavapor, and lipid films were rehydrated with buffer

$110(50 \mathrm{mM} \mathrm{NaP}, 150 \mathrm{mM} \mathrm{NaCl}, 0.2 \mathrm{mM}$ EDTA, $\mathrm{pH} 7.5)$ by warming the lipid film at $40^{\circ} \mathrm{C}$ and vortexing, rendering a final total lipid concentration of $1 \mathrm{mM}$. Large unilamellar vesicles were prepared by mechanical extrusion using a $10-\mathrm{mL}$ Thermobarrel extruder (LipexTM. Northerns Lipids Inc.). The lipid suspension was extruded repeatedly (10 times) through a $100 \mathrm{~nm}$ polycarbonate filter at $40^{\circ} \mathrm{C}$. To verify the appropriate size of the liposomes, the mean diameter was checked using Dynamic Light Scattering (Zetasizer Nanoseries S, Malvern instruments).

\section{Direct SPR binding assay}

SPR experiments were carried out in a Biacore 100 instrument from GE Healthcare. The SPR chip (a 2D carboxymethyldextran surface) from Xantec was modified by covalent attachment of phytosphingosine (TebuBio) to allow the capture of neutral or negatively charged liposomes containing DMPC or DMPC-DMPG (2:1), respectively.

120 Experiments were carried out at $298 \mathrm{~K}$ where these lipids and lipid mixtures are in the liquid crystalline phase. Control experiments with the equivalent unsaturated oleyl lipids DOPC-DOPG (2:1) with a transition temperature of $256 \mathrm{~K}-256$ $\mathrm{K}$ gave very similar results (Figure S6). The reference channel was blocked with BSA, with a $1 \mathrm{mg} / \mathrm{ml}$ injection at $10 \mu \mathrm{l} / \mathrm{min}$ for $100 \mathrm{~s}$. The immobilization protocol and subsequent liposome coating were performed in the instrument chamber, at a flow rate of $10 \mu \mathrm{l} / \mathrm{min}$. To minimize mass transport phenomena, a flow rate of $50 \mu 1 / \mathrm{min}$ was used to

125 monitor the interaction of MyrUSH3 with liposomes. The SPR response of MyrUSH3 binding to liposomes was followed for $60 \mathrm{~s}$ (association phase), while the subsequent washing lasted $200 \mathrm{~s}$ (dissociation phase). Protein concentration ranged from $1 \mu \mathrm{M}$ to $20 \mu \mathrm{M}$, and all experiments were performed in the running buffer, which comprised $50 \mathrm{mM} \mathrm{NaP}, 150 \mathrm{mM} \mathrm{NaCl}$ and $0.02 \mathrm{mM}$ EDTA, $\mathrm{pH} 7.5$, unless indicated otherwise. The surface was regenerated with a $10-\mathrm{mM} \mathrm{HCl}$ pulse followed by a $20-\mathrm{mM}$ CHAPS pulse, both for $30 \mathrm{~s}$ at $10 \mu 1 / \mathrm{min}$. Each experiment was started with

130 freshly captured liposomes. The liposome coating itself was reproducible, reaching an average value of 6785 RU (standard deviation $625 \mathrm{RU}$ ) for DMPC liposomes, and $8193 \mathrm{RU}$ (standard deviation $608 \mathrm{RU}$ ) for DMPC:DMPG liposomes, guaranteeing reproducible protein responses as shown in Figure 4.

\section{SPR detected antibody capture}

135 MyrUSH3 dissociated very quickly from liposomes, but the presence of a residual population of lipid bound MyrUSH3 was detected by the SPR response observed after injecting antiSH4 antibody (Abcam) at a 1:10 dilution in the running buffer. The association and dissociation kinetics of these PB species were studied separately: dissociation was measured by recording the antibody response at increasing times after a constant association time of $60 \mathrm{~s}$, while association was monitored by detecting the antibody response at a fixed time after the end of increasingly long association times. The

140 flow rate was kept at $50 \mu \mathrm{l} / \mathrm{min}$ during protein association and dissociation, and decreased to $30 \mu 1 / \mathrm{min}$ just before antibody injection.

\section{Model and data analysis}

Direct SPR responses of MyrUSH3 binding to liposomes were analyzed using the Biaevaluation software from Biacore using two alternative models: the 1:1 model and the conformational change model. Data of the PB fraction, obtained by antiSH4 antibody secondary detection, were analyzed using Igor software, with the models described in the results section. All data were double referenced (reference channel and baseline subtraction). 
Direct SPR data from MyrUSH3 binding to liposomes were initially fitted with a simple 1:1 Langmuir model described by reaction 1 .

$\mathrm{P}+\mathrm{L} \quad \Leftrightarrow \quad \mathrm{PL} \quad\left(\mathrm{k}_{\mathrm{a}}\right.$ and $\left.\mathrm{k}_{\mathrm{d}}\right) \quad$ reaction $(1)$

where P represents the MyrUSH3 protein, L the lipids, and PL the lipid-bound protein, the latter forming with the association and dissociation rate constants $\mathrm{k}_{\mathrm{a}}$ and $\mathrm{k}_{\mathrm{d}}$ respectively.

The SPR response is proportional to [PL], obeying the equation:

$$
[P L](t)=L_{e q} \cdot\left(1-\exp \left(-\left(k_{\mathrm{a}} \cdot[\mathrm{P}]+\mathrm{k}_{\mathrm{d}}\right) \cdot \mathrm{t}\right)\right)
$$

with $\mathrm{L}_{\mathrm{eq}}=\mathrm{L}_{\max }\left(\mathrm{k}_{\mathrm{a}} \cdot[\mathrm{P}] /\left(\mathrm{k}_{\mathrm{a}} \cdot[\mathrm{P}]+\mathrm{k}_{\mathrm{d}}\right)\right)$

The amount of immobilized liposomes limits the highest possible protein response, proportional to $\mathrm{L}_{\max }$. The rate constants can be estimated by globally fitting the binding curves at various concentrations. The association constant $\mathrm{K}_{\mathrm{a}}$ can be obtained from the ratio of rate constants $\mathrm{K}_{\mathrm{a}}=\mathrm{k}_{\mathrm{a}} / \mathrm{k}_{\mathrm{d}}$.

$\mathrm{K}_{\mathrm{a}}$ can also be obtained by fitting the equilibrium SPR responses $\left(\mathrm{L}_{\mathrm{eq}}\right)$ obtained at various protein concentrations [P]. In the 1:1 Langmuir model, the relevant equation is a modification of equation (2):

$$
\mathrm{L}_{\mathrm{eq}}=\mathrm{L}_{\max }\left([\mathrm{P}] /\left([\mathrm{P}]+1 / \mathrm{K}_{\mathrm{a}}\right)\right)
$$

\section{Results}

MyrUSH3 contains the first three domains of c-Src: the polybasic peptide SH4, the intrinsically disordered Unique Domain and the SH3 domain (Figure 1). The SH4 domain is myristoylated at its N-terminal and is the primary membrane-anchoring point of c-Src. The Unique and SH3 domains contain additional lipid binding regions [24]. MyrUSH3 was obtained in E. coli cells by co-expression with yeast N-Myristoyl Transferase. Figure 1a shows the

170 domain structure of full-length Src and Figure 1b the amino acid sequence of the USH3 construct used in this study, highlighting the three lipid binding regions SH4, Unique Lipid Binding Region (ULBR) and the RT loop in the SH3 domain.

\section{Persistent binding of MyrUSH3 to liposomes}

175 MyrUSH3 binding to immobilized DMPC, DMPC:DMPG or DOPC:DOPG liposomes was followed by SPR at 298K. These lipid systems are in the fluid phase under these conditions. This approach consisted of the following steps: i) liposome immobilization; ii) injection of MyrUSH3 (association phase); iii) washing with a protein-free buffer (dissociation phase); iv) antiSH4 antibody injection; and v) surface regeneration by washing away the liposomes. A complete cycle of MyrUSH3 binding to DMPC:DMPG liposomes is shown in Supplementary Material (Figure S.1).

180 The association phase revealed rapid binding and equilibrium was reached quickly. The response curve also revealed a rapid dissociation. However, the baseline did not return to its original value (the liposome capture value), but remained stable at a slightly higher level, thereby indicating the presence of PB MyrUSH3 proteins that were not removed by washing with the running buffer. Due to the small population of PB species, a secondary detection was used to confirm their presence and to quantify them. In this regards, $350 \mathrm{~s}$ after the beginning of the dissociation phase, antiSH4

185 antibody was injected over $60 \mathrm{~s}$. As a control, the antibody was also injected over protein free liposomes. In this case, no response was observed: MyrUSH3 and antiSH4 antibody were completely removed with the lipids when applying the regeneration conditions as described in the method section.

\section{Electrostatic and hydrophobic components of MyrUSH3 binding to DMPC:DMPG liposomes.}

190 The myristoylated N-terminal region of c-Src is known to be essential for binding but the PB population had not previously been reported. In order to check whether persistent binding was caused by the presence of additional lipid binding outside the myristoylated SH4 domain, we measured the binding of a synthetic peptide, containing only residues 2-16 of c-Src with a myristoyl group attached at its N-terminus (MyrSH4), to liposomes. The presence of a PB MyrSH4 population was clearly observed by injection of antiSH4 antibody (Figure 2a). The affinity of the antibody for

195 the two SH4 containing molecules may not be directly comparable, impairing comparison of the relative antibody responses of MyrSH4 or MyrUSH3 PB species. Nevertheless, we can still conclude that formation of the PB species is a property of the MyrSH4 domain and does not require the presence of the additional binding regions in the Unique and SH3 domains. The decrease of the direct SPR response curve during dissociation was slower in MyrSH4 than in MyrUSH3, thereby suggesting that the presence of the secondary lipid binding domains in USH3 enhances the 
200 dissociation rate of the MyrSH4 region from the membrane. This apparently contradictory result may be explained by the recently reported interaction between the SH4 and SH3 domains of c-Src [25]. Since MyrUSH3 provides a more native-like environment and gives a stronger SPR response than MyrSH4, we focused our study on acylated USH3. The effect of myristoylation on the capacity of c-Src to bind membranes has been extensively discussed. It was concluded that the binding energy provided by the insertion of a single myristoyl chain was not sufficient to explain the stable

205 insertion of c-Src in the membrane. However, the electrostatic interaction between the positively charged SH4 domain and negatively charged lipids, together with myristoyl chain insertion, was enough to ensure stable binding [26] [27] [28]. In order to explore the contribution of the hydrophobic and electrostatic components, we first compared the binding of MyrUSH3 and LaurUSH3 to liposomes. These two species differ only in the length of the N-terminal acyl chain with lauric acid having a 12-carbon chain and myristic acid a14-carbon chain.

210 Figure $2 \mathrm{~b}$ compares the binding of MyrUSH3 $(1 \mu \mathrm{M}$ and $20 \mu \mathrm{M})$ and LaurUSH3 $(20 \mu \mathrm{M})$ to DMPC:DMPG liposomes. When the two proteins were injected at the same concentration $(20 \mu \mathrm{M})$, the direct SPR response of LaurUSH3 was circa four times lower than that of MyrUSH3, and the secondary detection by antiSH4 antibody gave barely any response for LaurUSH3. In comparison, the injection of $1 \mu \mathrm{M}$ MyrUSH3, which provided a direct SPR response half that of the $20 \mu \mathrm{M}$ LaurUSH3 injection, still gave a clearly detectable antibody response. These experiments show that

215 while shortening of the acyl chain clearly reduced, but did not eliminate, the binding of the fast dissociating form, it abolished the formation of PB species.

The effect of the electrostatic interactions on the formation of PB MyrUSH3 species was first tested by comparing MyrUSH3 $(10 \mu \mathrm{M})$ binding to neutral (DMPC) or charged (DMPC:DMPG 2:1) liposomes. For negatively charged liposomes, the direct SPR response was higher, the dissociation was slower and the baseline recovered after washing

220 was also higher. Secondary detection confirmed that the population of the PB forms was higher for MyrUSH3 bound to negatively charged lipids (Figure 2c). The electrostatic interaction of MyrUSH3 with charged liposomes increased at lower ionic strengths (Figure 2d). The direct SPR response was doubled when the salt concentration was reduced from $150 \mathrm{mM}$ to $0 \mathrm{mM}$, in $50 \mathrm{mM} \mathrm{NaP}$. A markedly slower dissociation was observed at low ionic strength. Consistently, we observed a much stronger response to the antibody, about four times larger, thereby suggesting that the relative

225 population of the PB forms with respect to rapidly dissociating ones is increased at low ionic strength. However, the decrease in the baseline to values lower than before the MyrUSH3 injection suggests that at low ionic strength, MyrUSH3 binding affects the immobilized liposomes. Consequently, we quantitatively analyzed the two binding processes presented below in the presence of $150 \mathrm{mM} \mathrm{NaCl}$, a value close to physiological ionic strength.

\section{Kinetics analysis of the direct response of MyrUSH3 binding to liposomes}

MyrUSH3 binding was initially measured at protein concentrations ranging from $1 \mu \mathrm{M}$ to $100 \mu \mathrm{M}$ using the direct SPR response. Below $20 \mu \mathrm{M}$, the binding curves had the expected shape, with an association phase leading to a plateau when reaching equilibrium, followed by protein dissociation after washing with buffer. At concentrations higher than $20 \mu \mathrm{M}$, the binding curves did not reach a stable plateau after $60 \mathrm{~s}$. Failure to reach a stable equilibrium, at high concentration may be due to slow binding or a cooperative binding of peptides over the lipid surface [30] - see Supplementary Materials, Figure S.2a. We chose to restrict our study to concentrations between $1 \mu \mathrm{M}$ and $20 \mu \mathrm{M}$.

The direct response of MyrUSH3 binding to liposomes was studied in order to obtain kinetics and affinity information on the fast associating and dissociating population. While the presence of multiple binding modes would in principle prevent the use of a simple model, the contribution of the PB form to this response was very small. We thus first analyzed the data with a simple 1:1 Langmuir model. From a formal point of view, peptides partition to liposomes, and stoichiometry is most often unknown. Nevertheless, simplification using the Langmuir model gives a satisfactory starting point for analyzing kinetics data obtained by SPR [31]. A reasonable fit of our experimental curves was obtained, especially for the association phase, although deviations were observed at the end of the dissociation phase. (see Supplementary Materials Figure S.2b). The association constant derived from the 1:1 Langmuir model $\left(1.5410^{4} \pm\right.$

$\left.2457.0110^{3} \mathrm{M}^{-1}\right)$ for the binding of MyrUSH3 to DMPC liposomes is in good agreement with the value $\left(9^{*} 10^{3} \mathrm{M}^{-1}\right)$ obtained by Buser et al. for MyrSH4 peptide using partition methods [27]. Binding to charged liposomes occurs with circa one order of magnitude higher affinity $\left(1.910^{5} \pm 6.5210^{4} \mathrm{M}^{-1}\right)$, confirming the electrostatic contribution to the binding affinity. Kinetic and binding constants are given in Table S2 of the Supplementary Material.

An improved fitting of the dissociation phase could be obtained with a conformational change model in the 250 Biaevaluation software, which assumes two separate bound forms. Figure S.2b shows expansions comparing the experimental and best-fit curves for MyrUSH3 binding to neutral and charged liposomes using the two models. While the conformational change model improves the fitting of the (fast) processes that dominate the direct SPR response and points to the presence of more than one second form of bound MyrUSH3, the corresponding dissociation rates are too fast to explain the PB form observed after more than $60 \mathrm{~s}$ of dissociation.

255 The existence of a persistently bound form of c-Src has strong biological implications and the characterization of the 
nature and kinetics properties of MyrUSH3 is thus of great interest.

\section{Kinetics analysis of the PB form of MyrUSH3 binding to liposomes}

260 The observation of the PB form after extensive washing, a procedure that removed the fast dissociating population, offers the possibility to study association and dissociation kinetics of the two species independently. In addition, secondary detection by antiSH4 antibody capture provided the required sensitivity to study the binding kinetics of the PB form, which is much less populated and gives a low SPR signal.

By varying the MyrUSH3 association time, and applying the antibody at a constant time after the association phase, we were able to follow the association kinetics of the PB form independently. In a similar manner, by keeping constant the association time of MyrUSH3 binding to liposomes, and applying the antibody at different times of the dissociation phase, we were able to independently study its dissociation. The experimental design is shown in Figure 3.

Figure $4 \mathrm{a}$ and $4 \mathrm{~b}$ show the experimental curves obtained by this method for a given association/dissociation time (60 $\mathrm{s} / 350 \mathrm{~s}$ ). The antibody solution was injected at a constant dilution and therefore, the observed SPR response is assumed to reflect the population of MyrUSH3 present on the liposome surface. Indeed, the antibody response was greater with increasing the concentration of MyrUSH3 during the association phase.

Figure $4 \mathrm{c}$ and $4 \mathrm{~d}$ show the dissociation phase of the PB form of MyrUSH3 bound to neutral and charged liposomes. Dissociation from neutral liposomes is negligible in the observed time frame and very slow from charged liposomes. The population of MyrUSH3 that remains bound to neutral DMPC liposomes did not decrease between 400 and $7200 \mathrm{~s}$ after the end of the association phase. In the case of DMPC:DMPG liposomes, the bound population of MyrUSH3 after $400 \mathrm{~s}$ was higher than with neutral lipids and slowly decreased between 400 and $7200 \mathrm{~s}$. After $2 \mathrm{~h}$, the population of MyrUSH3 bound to neutral and charged liposomes reached a plateau, suggesting that a fraction of this form became irreversibly bound to both types of liposomes.

On the light of the above observations, the process leading to the appearance of the PB form was analyzed using a

280 "conformational change"-like model in which PL gives rise to PB in an irreversible manner. The rate constant of the transformation of PL to PB is designated as $\mathrm{k}_{\text {on }}$.

$\mathrm{P}+\mathrm{L} \Leftrightarrow \mathrm{PL} \quad\left(\mathrm{k}_{\mathrm{a}}\right.$ and $\left.\mathrm{k}_{\mathrm{d}}\right) \quad$ reaction $(1)$

$$
\mathrm{PL} \Rightarrow \mathrm{PB} \quad\left(\mathrm{k}_{\text {on }}^{\prime}\right) \quad \text { reaction }(2)
$$

The model is presented using generic lipid containing species [L]; however the actual stoichiometry of the interaction is not known. For this reason we write the derived equations in terms of the fraction of lipids bound to protein, $\theta$.

$\theta=[\mathrm{PL}] / \mathrm{L}_{\max }+[\mathrm{PB}] / \mathrm{L}_{\max }$

$1-\theta=[\mathrm{L}] / \mathrm{L}_{\max }$

Since the amount of PB formed is small compared to that of PL, we assume that the kinetics of the formation of PB does not modify the equilibrium of PL:

$$
\theta \sim[\mathrm{PL}] / \mathrm{L}_{\max }
$$

This hypothesis explains the (experimentally observed) dependence of the binding levels with protein concentration. Without this hypothesis, the maximum concentration of [PB] would be the same for all concentrations in our equations, due to the irreversibility of the second equation.

Assuming reaction (1) is at equilibrium, we can derive an expression of $\theta$.

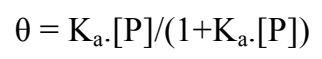

We want to solve the kinetics equation corresponding to reaction (2) 
In terms of occupation

$\mathrm{d} \theta_{\mathrm{PB}} / \mathrm{dt}=\mathrm{k}_{\mathrm{on}}^{\prime} \cdot \theta_{\mathrm{PL}} \quad$ eq. (9)

Resolution of equation (9) is detailed in Supplementary Material S.4. The following expression is obtained:

$\theta_{\mathrm{PB}}=\theta \cdot\left(1-\exp \left(-\mathrm{k}_{\mathrm{on}}^{\prime} \cdot \mathrm{t}\right)\right)$

320 We call R the SPR response of the antiSH4 antibody binding to PB, which is proportional to [PB].

$\mathrm{R}=\mathrm{C}_{1} \cdot\left(1-\exp \left(-\mathrm{k}_{\text {on }}^{\prime} \cdot \mathrm{t}\right)\right)$

with $\mathrm{C}_{1}$ proportional to $\theta$. $\mathrm{L}_{\max }$.

We fitted equation (11) locally to the experimental curves obtained at various concentrations. The best-fit values are displayed in Figure 5a for DMPC and Figure 5b for DMPC:DMPG. In the Supplementary Material, Table S.4 gives the values of the best-fit parameters.

We observe that k' on depends linearly on protein concentration (Figure 5c-5d).

This concentration dependence of the apparent rate constants for the conversion between the initially (and reversibly) bound form of MyrUSH3 to the PB form is an experimental indication that the conversion between the two forms of is a bimolecular process and suggests that the PB species of MyrUSH3 are, at least, dimeric forms.

335 Dimerization can be explicitly included in the binding model, assuming that PL can interact with a second P molecule to form $\mathrm{PB}$, which we now explicitly label as $\mathrm{PB}_{2}$ to stress its dimeric nature, with an association rate constant $\mathrm{k}_{\mathrm{on}}$ :

$\mathrm{P}+\mathrm{L} \Leftrightarrow \mathrm{PL} \quad\left(\mathrm{k}_{\mathrm{a}}\right.$ and $\left.\mathrm{k}_{\mathrm{d}}\right) \quad$ reaction $(1)$

$\mathrm{PL}+\mathrm{P} \Rightarrow \mathrm{PB}_{2} \quad\left(\mathrm{k}_{\mathrm{on}}\right) \quad$ reaction (4)

In this case, $\theta_{\mathrm{PB} 2}$ can be expressed as:

$\theta_{\mathrm{PB} 2}=\theta \cdot\left(1-\exp \left(-\mathrm{k}_{\mathrm{on}} \cdot[\mathrm{P}] . \mathrm{t}\right)\right)$

eq. (12)

Fitting using equation (11) is equally applicable but in this case

$\mathrm{k}_{\mathrm{on}}^{\prime}=\mathrm{k}_{\mathrm{on}} \cdot[\mathrm{P}]$

eq. (13)

350 The bimolecular $\mathrm{k}_{\text {on }}$ (unit $\mathrm{M}^{-1} \cdot \mathrm{s}^{-1}$ ) can be obtained from the slope of the linear fit between $\mathrm{k}_{\text {on }}^{\prime}$ and the protein concentration [P]. For DMPC liposomes, $\mathrm{k}_{\mathrm{on}}=1.2 * 10^{4} \mathrm{M}^{-1} \cdot \mathrm{s}^{-1}$, while for DMPC:DMPG liposomes, $\mathrm{k}_{\mathrm{on}}=5.1^{*} 10^{3} \mathrm{M}^{-1} \mathrm{~s}^{-1}$ The $\mathrm{k}_{\text {on }}$ value obtained with this model for neutral liposomes is larger than for charged ones. From the fitted $\mathrm{k}_{\text {on }}$ values it is possible to estimate and compare the true velocity of the PB species formation on both types of liposomes and at different protein concentrations. According to our model, the velocity $\mathrm{v}$ is given by:

$\mathrm{v}=\mathrm{k}_{\mathrm{on}}^{\prime} \cdot \mathrm{R}_{\mathrm{eq}}$

where $\mathrm{R}_{\mathrm{eq}}$ is the direct SPR response at equilibrium (in Response Units)

360 The ratio of velocities of PB species formation in DMPC:DMPG versus DMPC alone, plotted as a function of protein concentration $[\mathrm{P}]$, can be found in the Supplementary Material (Figure S.5). At low concentration, the formation is faster in charged liposomes, but the ratio reaches a plateau close to 1 at $10 \mu \mathrm{M}$ and higher concentrations, suggesting that the inherent rate of dimer formation is the same in both cases. The difference observed at low concentration may come from a higher concentration of bound monomers and/or the accumulation of free protein with their positively 365 charged N-terminal region oriented towards the surface.

The model presented is only a first approximation to the binding process, which is probably more complex. The bound species are assumed to be either fast dissociating monomers or PB forms. While the second order rate constant needed to fit the experimental rate of formation of the PB species strongly points to the formation of dimers, the presence of higher oligomers cannot be ruled out. Also the model assumes that the reaction (4) is irreversible, which would ultimately lead to an infinite concentration of $\mathrm{PB}_{2}$, since $\mathrm{P}$ is supplied in excess at a constant concentration. This is obviously not the case and the maximum concentration of $\mathrm{PB}_{2}$ must be limited, possibly by the increase in lateral 
pressure induced by the inserted proteins (or their mutual electrostatic repulsion), which reaches a maximum level beyond which no further protein monomer or dimer can be inserted.

\section{Discussion}

All the members of the SFK have a myristoyl group attached to their N-terminal glycine residues. In addition, all of them, with the exception of c-Src, contain additional palmitoyl group(s) attached to cysteine residues in their SH4 domain. The hydrophobic acyl groups are essential for anchoring SFKs to membranes. The rest of the protein remains 380 soluble and is connected to the membrane-anchored SH4 domain through the intrinsically disordered Unique domain.

In the case of c-Src, insertion of a single myristoyl group does not provide enough hydrophobic energy to ensure stable binding. This problem has been extensively discussed in the literature [14] [26][27][28] and the consensus view is currently that permanent anchoring of c-Src is achieved by the combination of hydrophobic insertion of the myristoyl group and electrostatic interaction of the strongly positively charged SH4 domain with negatively charged membranes.

385 Here we have used SPR to characterize the kinetics of binding of Myristoylated USH3, a construct that contains the main lipid-binding region of c-Src, to immobilized liposomes. Surprisingly, two kinetically well-defined binding events were observed. The first one has fast association and dissociation rate constants and can be followed directly by the SPR response of MyrUSH3 injections. At low protein concentrations, a standard analysis of the binding curves gives an association constant that is in good agreement with that obtained by partition methods in similar systems. Most of the

390 bound species can be washed away from the immobilized liposomes with buffer. However, a small population of a PB form of MyrUSH3 was observed. While the corresponding SPR response was only a small shift from baseline level, the presence of the PB species was confirmed by capture of an antiSH4 antibody by the proteins remaining on the liposome surface. The antibody SPR response is proportional to the PB population. The latter was retained for more than $2 \mathrm{~h}$ and could be eliminated only by detachment of the immobilized liposomes by means of detergents. When liposomes were 395 not exposed to protein, no antibody was captured.

A PB form was also observed using a synthetic myristoylated peptide containing only the SH4 domain (MyrSH4). In contrast, USH3 with a C12 lauryl chain instead of the C14 myristoyl chain showed no PB form, though fast association and dissociation processes were observed (with lower affinity than MyrUSH3). Thus from the structural point of view,

$400 \mathrm{MyrSH} 4$ is a necessary and sufficient requirement for the observation of persistent binding.

The electrostatic contribution was tested by comparing MyrUSH3 binding to neutral and negatively charged liposomes, and by the effect of the ionic strength. MyrUSH3 affinity and also the rate of formation of the PB form increased in negatively charged lipids. The effect is consistent with an electrostatically driven approach of MyrSH4 to the liposome surface.

405 The very slow dissociation kinetics and the secondary detection using antiSH4 antibody allowed an independent analysis of the kinetics of PB species formation. The association kinetics depends on the population of the labile form of bound MyrUSH3 and the concentration of free MyrUSH3 during the association phase. Thus, PB formation follows an effective second order law, thereby suggesting that the PB form contains more than one MyrUSH3 molecule.

410 While membrane-integrated receptor kinases are known to oligomerize, usually in response to ligand binding, and this process triggers signaling events, non-receptor kinases, like c-Src, are commonly believed to be essentially monomeric, even when anchored to membranes. However, c-Src phosphorylation by a second c-Src molecule is known to occur, therefore at least transient contacts between c-Src molecules are functionally important.

The kinetically detected self-association of MyrUSH3 on liposomes is in agreement with super resolution microscopy results with a construct containing the first 15 residues of the c-Src SH4 domain fused to photoswitchable (PS-GFP2) expressed in HeLa cells [32].

USH3 or its myristoylated form show no evidence of dimerization in solution, as verified in SAXS measurements (results not shown). Myristoylated c-Src SH4 fused to EGFP showed completely linear dependency of the fluorescence as a function of protein concentration on the range from $100 \mathrm{nM}$ to $5 \mu \mathrm{M}$, indicating no observable tendency to 420 aggregate in the absence of membranes (results not shown). Therefore, we conclude that the dimerization of MyrUSH3 is directly related to membrane binding. Figure 2 shows that replacing the $\mathrm{C} 14$ myristoyl chain by a C12 lauryl also abolished dimerization, although LaurUSH3 retains the capacity to interact with lipids albeit with a lower affinity. These results suggest that myristoyl insertion is essential for MyrUSH3 dimerization. The strict requirement for a myristoyl group suggests that the insertion of the fatty acid chain in the lipid bilayer is necessary for dimerization. We speculate that myristoyl insertion induces a conformation of the SH4 domain that allows the dimerization to occur. 
In addition, MyrUSH3 dimerization is observed in the presence of either neutral or negatively charged lipids and the dimer formed is irreversibly bound in both systems. The slow dissociation of MyrUSH3 dimers is consistent with the effectively irreversible binding of other SFKs in which two acyl chains are simultaneously inserted in the membrane.

The enhanced binding and dimer formation observed in negatively charged lipids probably reflects the additional binding affinity, which results in a larger concentration of bound MyrUSH3 monomer as well as the increased local concentration of soluble MyrUSH3 close to the membrane. Membrane tethered myristoylated IDPs can be considered to form a soft spherical particle around the charged liposomes [33].

The biological relevance of c-Src dimerization remains to be demonstrated but we suggest a possible functional role of the coexistence of a fast and a slow dissociation form of bound c-Src.

435 In its resting state, inactive c-Src is found predominantly in the perinuclear region of the cell. However, upon activation c-Src accumulates in specific regions of the membrane. Switching between monomeric and dimeric forms of c-Src would facilitate the fast exploration of the membrane surface until the target site is located, at which point the binding mode would switch to the persistent form and signaling would start. At low concentrations, myristoylated c-Src can probably attach and dissociate rapidly from the membrane surface, allowing fast diffusion on the 2D membrane surface and efficient exploration of large membrane areas. However, a local increase in c-Src concentration, for instance by high local concentrations of negatively charged lipids such as phosphatidyl inositide phosphates (PIPs), may result in the formation of c-Src dimers, which will remain in this particular membrane site and trans-activation will be facilitated, thus triggering the relevant signaling pathway.

A similar strategy is used by transcription factors to explore large regions of DNA. These systems switch from nonspecific low affinity binding mode, to retain the transcription factor close to the DNA surface and facilitate fast onedimensional diffusion, to specific, high affinity binding at the target site. The observed dual lipid binding mode of c-Src suggest a similar mechanism in which fast dissociation allow diffusion close to the $2 \mathrm{D}$ surface of the membrane, while dimerization fixes c-Src at the target site to initiate downstream signaling.

\section{Acknowledgments}

We thank Marta Taulés (CCiTUB) for technical help in the SPR experiments. We also thank Samira Jaeger, Irene Amata, Mariano Maffei, Enric Mayans Tayadella and Maria-Jesus Sanchez for their inputs. The bicistronic plasmid for the production of myristoylated proteins was a kind gift of Dieter Willbold. This work was partially supported by funds from MINECO (BIO2013-45793-R, FIS2013-41144-P and MAT2012-36270-C04-03), and la Fundació Marató TV3. ALLR holds an IRB/La Caixa fellowship.

\section{References}

[1] M.T. Brown, J.A. Cooper, Regulation, substrates and functions of Src, Biochim. Biophys. Acta. 1287:121-149 (1996).0

[2] S.M. Thomas, J.S. Brugge, Cellular functions regulated by Src family kinases. Annu. Rev. Cell Dev. Biol. 13:513-609 (1997).

[3] S.J. Parsons, J.T. Parsons, Src family kinases, key regulators of signal transduction. Oncogene. 23:7906-7909 (2004).

[4] M.C. Frame, Newest findings on the oldest oncogene; how activated Src does it. J. Cell Sci. 117:989-998 (2004).

[5] T.J. Yeatman, A renaissance for Src, Nat. Rev. Cancer 4:470-480 (2004).

[6] X.H. Zhang, Q. Wang, W. Gerald, C.A. Hudis, L. Norton, M. Smid, J.A. Foekens, J. Massagué, Latent Bone Metastasis in Breast Cancer Tied to Src-Dependent Survival Signals. Cancer Cell. 16:67-78 (2009).

[7] N.M. Han, S.A. Curley, G.E. Gatlick, Clin Cancer Res. Differential activation of pp60(c-src) and pp62(c-yes) in human colorectal carcinoma liver metastases. 2:1397-1404 (1996).

470 [8] H. Aligayer, D.D. Boyd, M.M. Heiss, E-K. Abdalla, S.A. Curley, G.E. Gatlick, Activation of Src kinase in primary colorectal carcinoma: an indicator of poor clinical prognosis. Cancer. 94:344-351 (2002).

[9] K.B. Kaplan, J.R. Swedlow, H.E. Varmus, D.O. Morgan, Association of p60c-src with endosomal membranes in mammalian fibroblasts. J. Cell Biol. 118:321-333 (1992).

[10] E. Sandilands, C. Cans, V.J. Fincham, V.G. Brunton, H. Mellor, G.C. Prendergast, J.C. Norman, G. Superti-Furga, M.C. Frame, RhoB and actin polymerization coordinate Src activation with endosome-mediated delivery to the membrane. Dev. Cell. 7:855-869 
(2004).

[11] R.L. Berdeaux, B. Diaz, L. Kim, G.S. Martin. Active Rho is localized to podosomes induced by oncogenic Src and is required for their assembly and function. J. Cell Biol. 166:317-323 (2004).

[12] J.F. Cote, and K. Vuori. GEF what? Dock180 and related proteins help Rac to polarize cells in new ways. Trends Cell Biol. 17(8):383-393 (2007).

[13] M.D. Resh, Myristylation and palmitylation of Src family members: the fats of the matter. Cell. 76:411-413 (1994).

[14] S. McLaughlin, A. Aderem, The myristoyl-electrostatic switch: a modulator of reversible protein-membrane interactions. Trends in Biochemical Sciences. 20(7):272-276 (1995).

[15] E. Sandilands, C. Cans, V. J. Fincham, V. G. Brunton, H. Mellor, G. C. Prendergast, J. C. Norman, G. Superti-Furga, M. C. Frame, RhoB and Actin Polymerization Coordinate Src Activation with Endosome-Mediated Delivery to the Membrane. Developmental Cell. 7:855-869 (2004).

[16] T. David-Pfeuty, S. Bagrodia, D. Shalloway, Differential localization patterns of myristoylated and nonmyristoylated c-Src proteins in interphase and mitotic c-Src overexpresser cells. Journal of Cell Science. 105:613-628 (1993).

[17] M. Donepudi, M. D. Resh, c-Src trafficking and co-localization with the EGF receptor promotes EGF ligand-independent EGF receptor activation and signaling. Cellular Signalling 20:1359-1367 (2008).

[18] J.E. Buss, M.K. Kamps, B.M. Sefton, Myristic acid is attached to the transforming protein of Rous sarcoma virus during or immediately after synthesis and is present in both soluble and membrane-bound forms of the protein. Molecular and Cellular Biology, 4(12):2697-2704 (1984).

495 [19] J.S. Goodwin, K.R. Drake, C. Rogers, L. Wright, J. Lippincott-Schwarts, M.R. Philips, A.K. Kenworthy. Depalmitoylated Ras traffics to and from the Golgi complex via nonvesicular pathway. J. Cell Biol. 170:261-272 (2005).

[20] E. Csapó, F. Bogár, Á. Juhász, D. Sebők, J. Szolomájer, G.K. Tóth, Z. Majláth, L. Vécsei, I. Dékány Determination of binding capacity and adsorption enthalpy between Human Glutamate Receptor (GluR1) peptide fragments and kynurenic acid by surface plasmon resonance experiments. Part 2: Interaction of GluR1270-300 with KYNA. Colloids and Surfaces B. Biointerfaces., 133: 6672 (2015).

[21] V. Hodnik, G.Anderluh. Surface plasmon resonance for measuring interactions of proteins with lipid membranes. Methods Mol Biol. 974:23-36. (2013).

[22] J. Zhang, R. Li, F.-L. Jiang, B. Zhou, Q.-Y. Luo, Q.-L.-Y. Yu, X.-L. Han, Y. Lin, H. He, Y. Liu, Y.-L. Wang. An electrochemical and surface plasmon resonance study of adsorption actions of DNA by Escherichia coli. Colloids Surfaces B.

505 Biointerfaces 117: 68-74 (2014).

[23] J.M. Glück, S. Hoffmann, B.W. Koenig, D. Willbold. Single Vector System for Efficient N-myristoylation of Recombinant Proteins in E. coli. PLoSONE 5(4): e10081. doi:10.1371/journal.pone.0010081 (2010).

[24] Y. Pérez, M. Maffei, A. Igea, I. Amata, M. Gair, A.R. Nebreda, P. Bernadó, M. Pons, Lipid binding by the Unique and SH3 domains of c-Src suggests a new regulatory mechanism. M. Sci Rep. 3:1295 (2013).

510 [25] M. Maffei, M. Arbesú, A.L. Le Roux, I. Amata, S. Roche, M. Pons, The SH3 Domain Acts as a Scaffold for the N-Terminal Intrinsically Disordered Regions of c-Src. Structure 23:893-902 (2015).

[26] C.A. Buser, C.T. Siga, M. D. Resh, S. McLaughlin, Membrane Binding of Myristylated Peptides Corresponding to the NH2 Terminus of Src. Biochemistry. 33:13093-13101 (1994).

515 [27] Electrostatics and the Membrane Association of Src: Theory and Experiment, Biochemistry 37: 2145-2159 (1998).

[28] D. Murray, L. Hermida-Matsumoto, C.A. Buser, J. Tsang, C.T. Sigal, N. Ben-Tal, B. Honig, M. D. Resh, S. McLaughlin, Amino-terminal basic residues of Src mediate membrane binding through electrostatic interaction with acidic phospholipids. Proc. Nati. Acad. Sci. USA, 91:12253-12257 (1994).

[29] S.H. White, W.C. Wimley, A. Lexey, S. Ladokhin, K. Khristova. Protein Folding in Membranes: Determining Energetics of Peptide-Bilayer Interactions

[30] G. Beschiaschvili, J. Seelig, Peptide Binding to Lipid Bilayers. Binding Isotherms and r-Potential of a Cyclic Somatostatin Analogue. Biochemistry 29:10995-11000 (1990).

525 [31] A. Gohlke, G. Triola, H. Waldmann, R. Winter, Influence of the Lipid Anchor Motif of N-Ras on the Interaction with Lipid Membranes: A Surface Plasmon Resonance Study. Biophysical Journal 98:2226-2235 (2010).

[32] D.M. Owen, C. Rentero, J. Rossy, A. Magenau, D. Williamson, M. Rodriguez, K. Gaus, PALM imaging and cluster analysis of protein heterogeneity at the cell surface. J. Biophoton. 3(7): 446-454 (2010).

[33] K. McDaniel, F. Valcius, J. Andrews, S. Das Electrostatic potential distribution of a soft spherical particle with a charged core and pH-dependent charge density Colloids Surfaces B. Biointerfaces, 127, 143-147 (2015). 


\section{Figure Captions}

Figure 1 Domain structure of $c-S r c$.

(a) Domain structure of c-Src. Lipid interacting regions (SH4, ULBR, RT loop) are highlighted. (b) MyrUSH3 Sequence. Residues 1 to 85 are intrinsically disordered and include the SH4 and Unique domains. Residues 88-150 correspond to SH3 domain and 151156 to the His6Tag.

Figure 2 Binding of myristoylated and laurylated peptides to liposomes.

(a) Comparison of binding of $50 \mu \mathrm{M}$ MyrSH4 and $100 \mu \mathrm{M}$ MyrUSH3 to liposomes. DOPC:DOPG 2:1 liposomes, in 50 mM NaP, $150 \mathrm{mM} \mathrm{NaCl}$.(b) Comparison of the binding of $20 \mu \mathrm{M}$ LaurUSH3 with that of $1 \mu \mathrm{M}$ and 20 $\mu M$ MyrUSH3. DMPC:DMPG 2:1 liposomes, in 50mM NaP and $150 \mathrm{mM} \mathrm{NaCl}$. (c) Binding of $10 \mu \mathrm{M} \mathrm{MyrUSH3}$ to DMPC or DMPC: DMPG 2:1 liposomes in 50mM

$545 \mathrm{NaP}$ and $150 \mathrm{mM} \mathrm{NaCl}$ (d) Binding of $2 \mu \mathrm{M} \mathrm{MyrUSH3}$ to DMPC:DMPG 2:1 liposomes in the presence or absence of $150 \mathrm{mM}$ $\mathrm{NaCl}$. In (a-d), the first curve corresponds to direct SPR peptide response, while the second is that of the secondary antibody detection.

550 Figure 3 Experimental scheme for the kinetics study of persistent forms of MyrUSH3.

(a) The build-up of antibody detectable MyrUSH3 over liposomes is monitored over time by varying the association time using a constant dissociation time. (b) Dissociation is followed by varying the dissociation time after a constant association time.

Figure 4 SPR sensograms and indirectly detected dissociation curves of persistent forms of MyrUSH3.

Direct and antibody detected SPR responses of 60s/350s association/dissociation of MyrUSH3 (between $2 \mu M$ and 20 $\mu M$ ) on (a) $D M P C$ and (b) DMPC:DMPG liposomes respectively. Dissociation from (c) DMPC and (d) DMPC:DMPG, detected by antibody secondary detection, following $60 \mathrm{~s}$ MyrUSH3 association time and variable dissociation times. *Baseline corresponds to an identical experiment in which the protein was omitted.

Figure 5 Indirectly detected binding curves of persistent forms of MyrUSH3.

Build-up of persistent MyrUSH3 on (a) DMPC and (b) DMPC:DMPG, detected by antibody secondary detection, using a constant dissociation time of $350 \mathrm{~s}$. The continuous curves correspond to the best-fits of the equation shown. Linear concentration dependence of $k_{\text {on }}^{\prime}$. for binding to (c) DMPC and (d) DMPC: DMPG liposomes. *Baseline corresponds to an identical experiment in which the protein was omitted. ** Duplicated results at $10 \mu M$ are shown; the error bar is shown in (c) and (d).

\section{Figures}


(a)

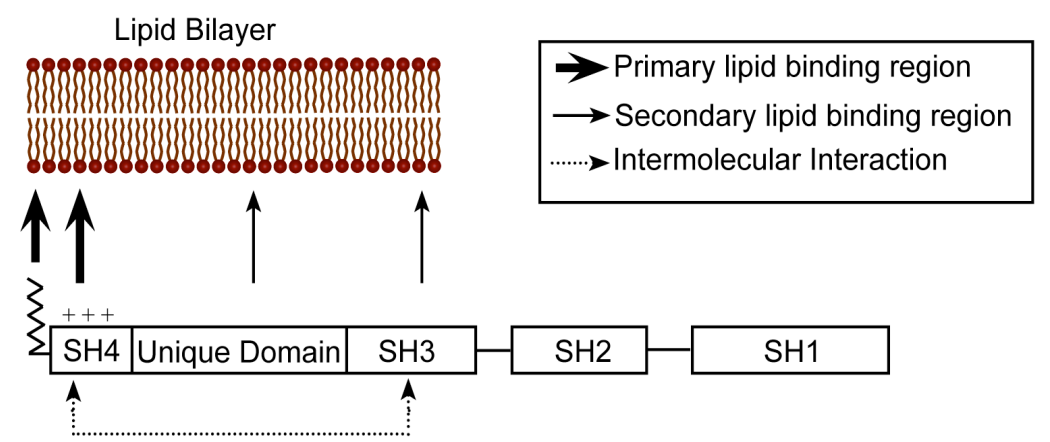

(b)

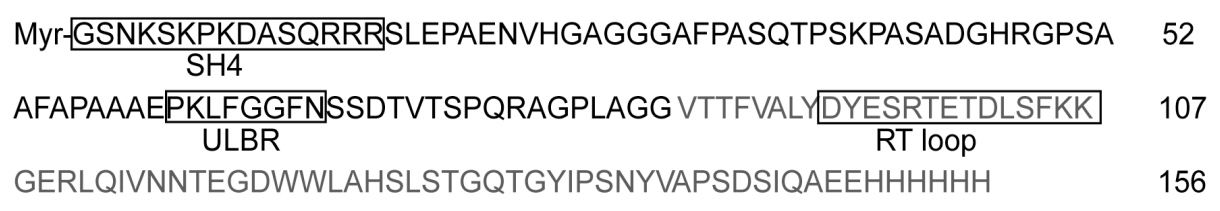

Figure 1 Domain structure of c-Src.

(a)

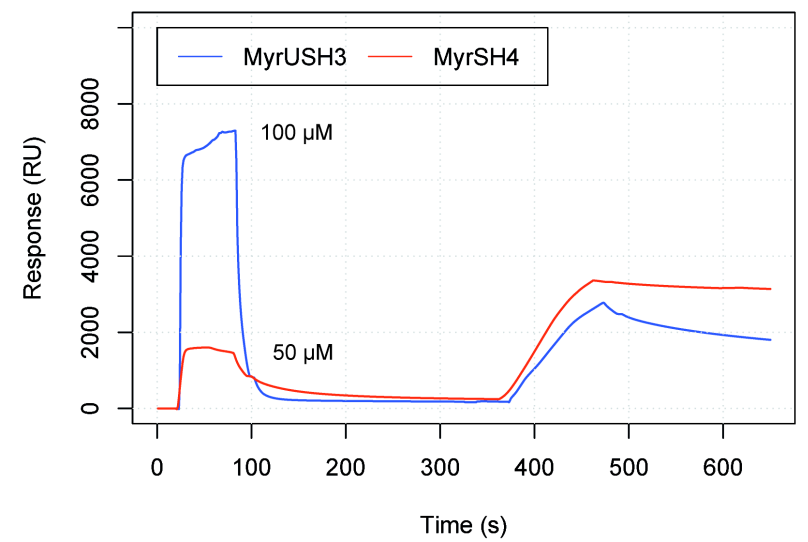

(c)

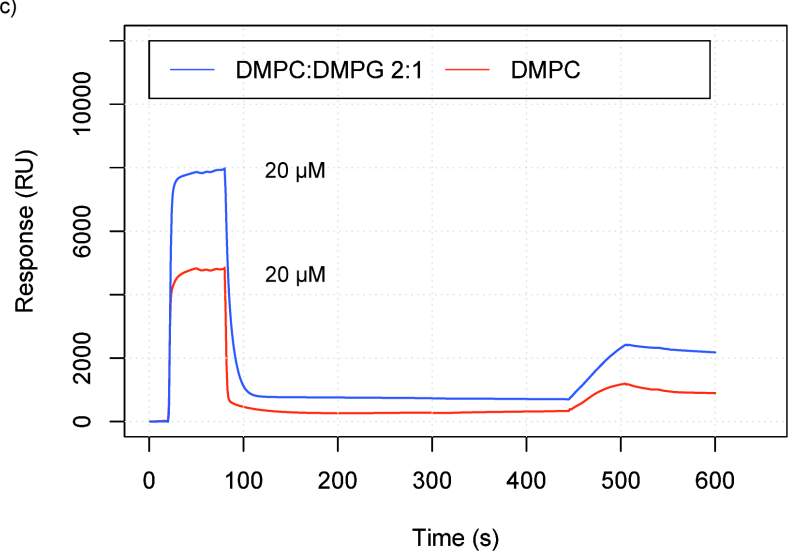

(b)

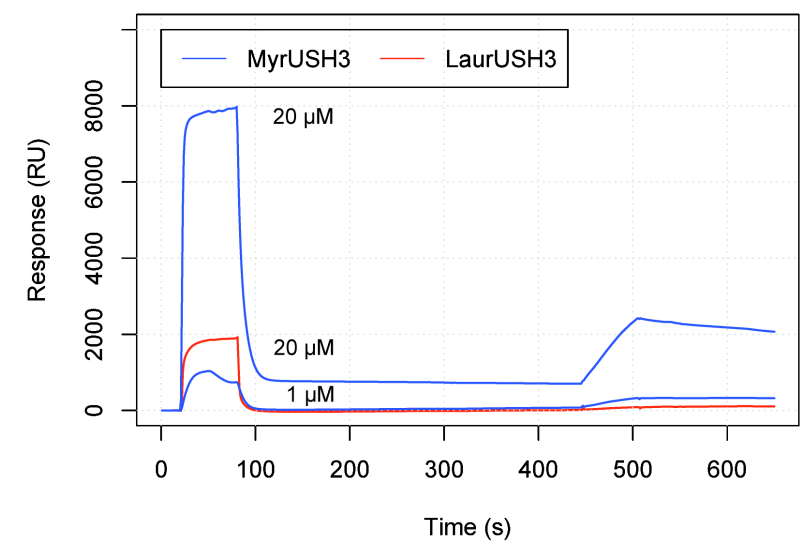

(d)

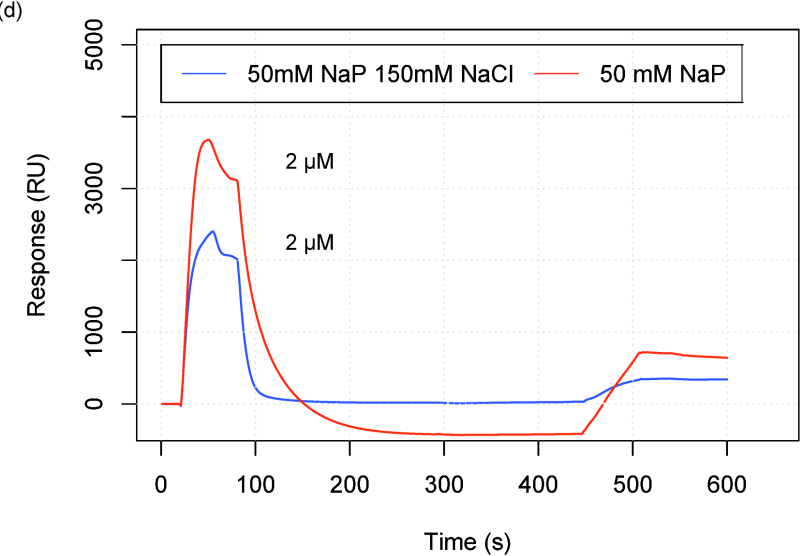

Figure 2 Binding of myristoylated and laurylated peptides to liposomes. 

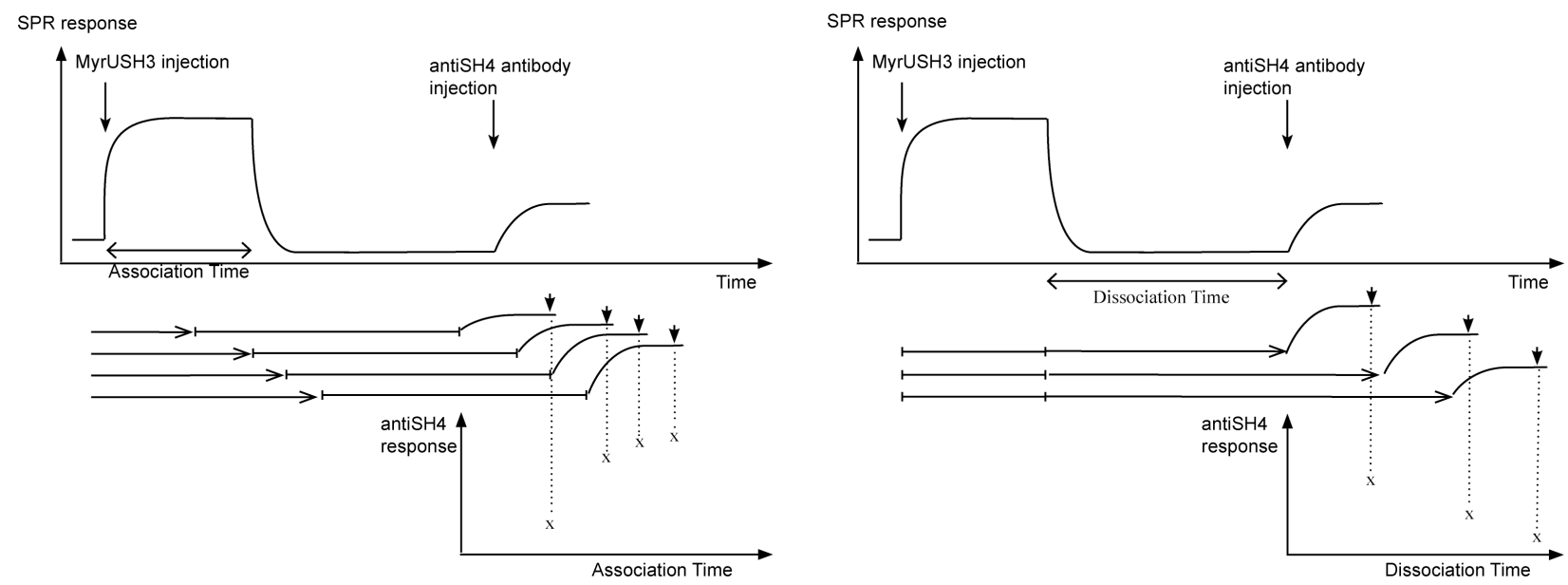

Figure 3 Experimental scheme for the kinetics study of persistent forms of MyrUSH3.

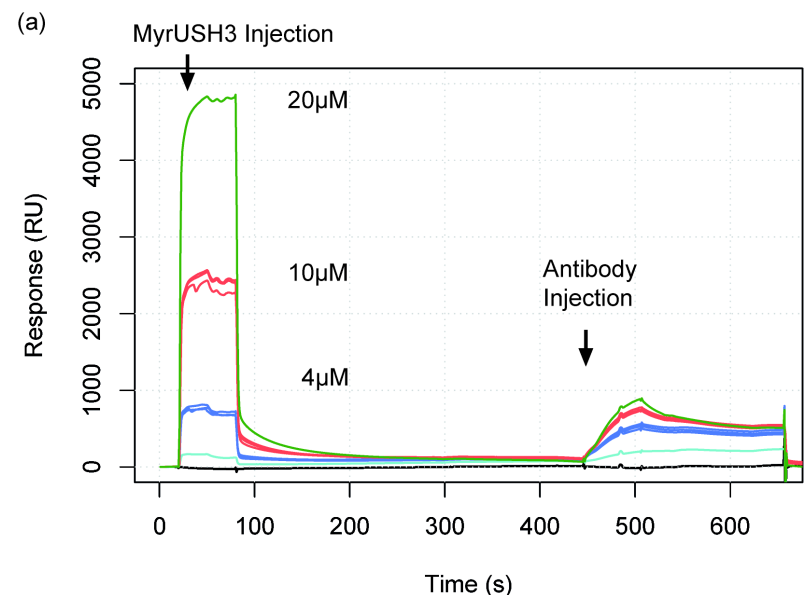

(b)

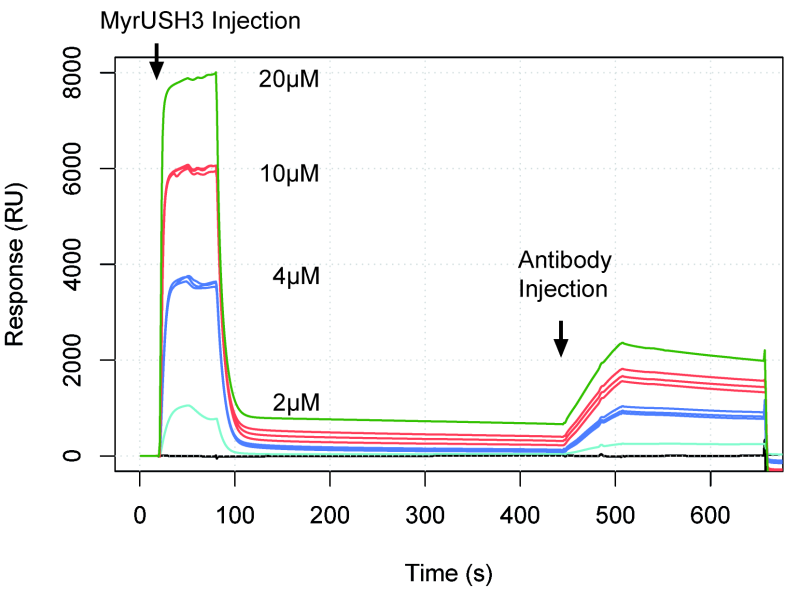

(c)

(d)
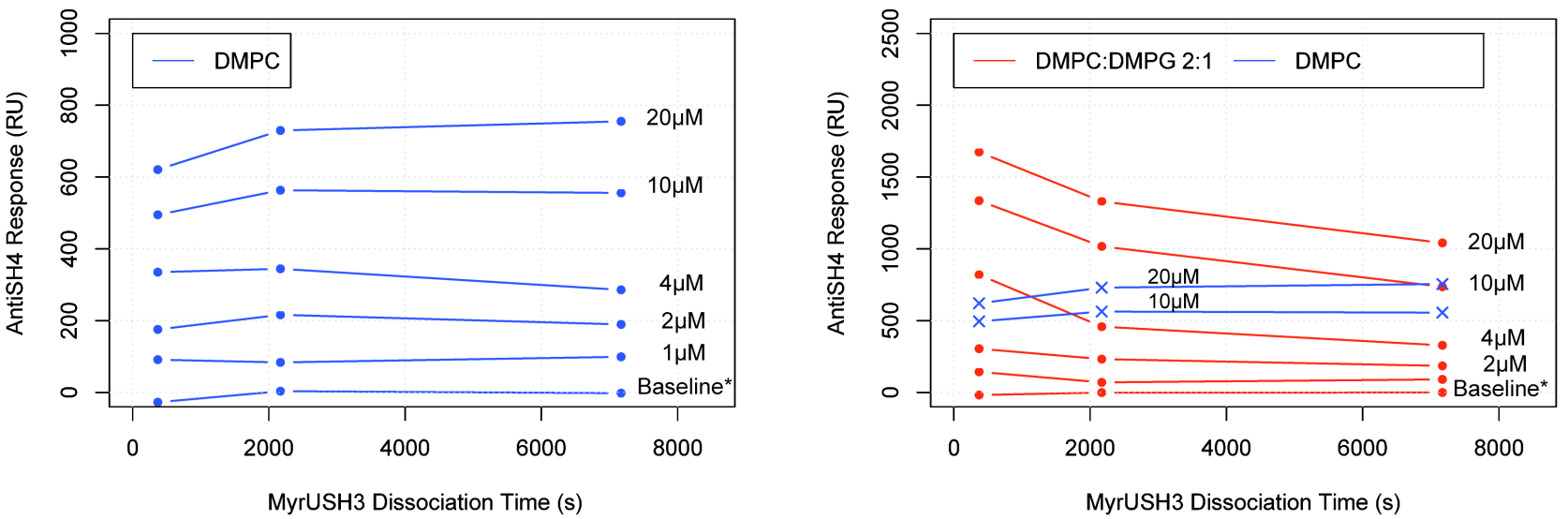

Figure 4 SPR sensograms and indirectly detected dissociation curves of persistent forms of MyrUSH3. 
(a)

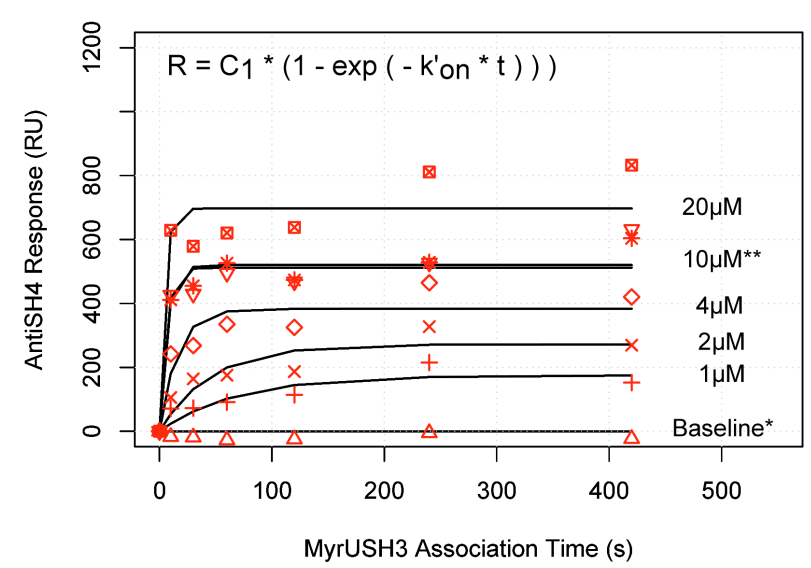

(c)

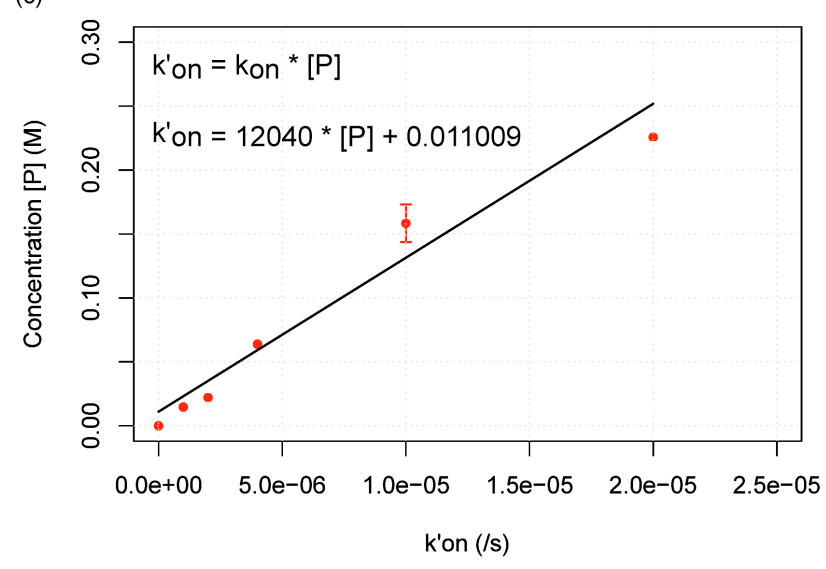

(b)

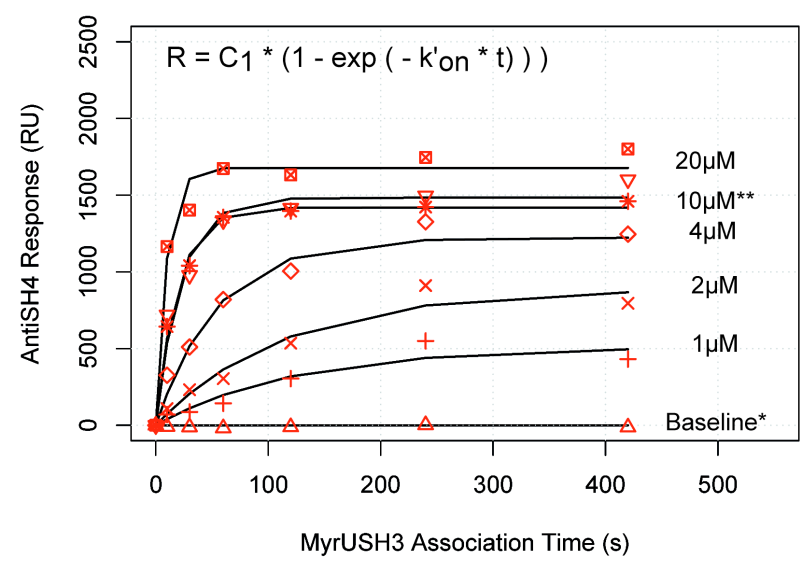

(d)

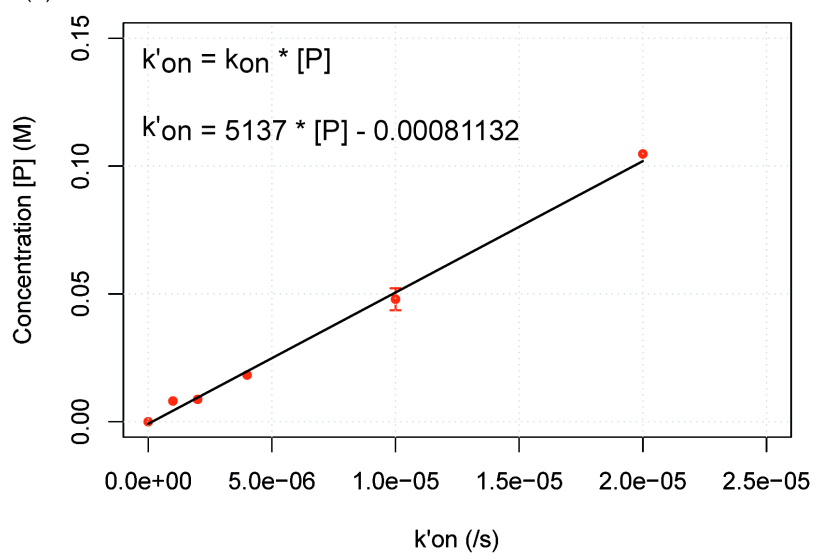

Figure 5 Indirectly detected binding curves of persistent forms of MyrUSH3.

600 